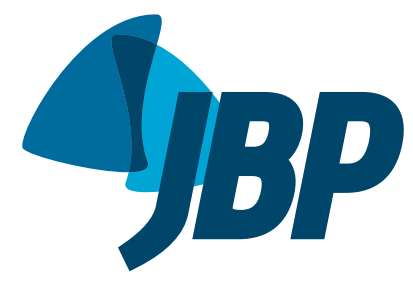

\title{
Robotic thoracic surgery for non-small cell lung cancer: initial experience in Brazil
}

\author{
Ricardo Mingarini Terra ${ }^{1,2,3,4, a}$, Benoit Jacques Bibas ${ }^{1,3,4, b}$, Rui Haddad ${ }^{5,6, c}$, \\ José Ribas Milanez-de-Campos ${ }^{1,3, d}$, Pedro Henrique Xavier Nabuco-de-Araujo 1,2,4,e, \\ Carlos Eduardo Teixeira-Lima ${ }^{5,6, f}$, Felipe Braga dos Santos ${ }^{5,6,9,}$, \\ Leticia Leone Lauricella $a^{1,2,4, h}$, Paulo Manuel Pêgo-Fernandes ${ }^{1,2,3, i}$
}

1. Disciplina de Cirurgia Torácica, Instituto do Coração, Hospital das Clínicas, Faculdade de Medicina, Universidade de São Paulo, São Paulo (SP) Brasil.

2. Hospital Sírio-Libanês, São Paulo (SP) Brasil.

3. Hospital Israelita Albert Einstein, São Paulo (SP) Brasil.

4. Instituto do Câncer do Estado de São Paulo - ICESP - Hospital das Clínicas Faculdade de Medicina, Universidade de São Paulo, São Paulo (SP) Brasil.

5. Hospital Copa Star - Rede D'Or, Rio de Janeiro (RJ) Brasil.

6. Pontifícia Universidade Católica do Rio de Janeiro, Rio de Janeiro (RJ) Brasil.

a. (iD) http://orcid.org/0000-0001-8577-8708

b. (ID) http://orcid.org/0000-0002-5092-0505

c. (iD) http://orcid.org/0000-0002-1288-3539

d. (iD) http://orcid.org/0000-0002-2385-7707

e. (DD http://orcid.org/0000-0003-0817-8180

f. (D) http://orcid.org/0000-0003-0953-1839

g. (iD http://orcid.org/0000-0001-6438-5665

h. (iD) http://orcid.org/0000-0002-8378-7704

i. (D) http://orcid.org/0000-0001-7243-5343

Submitted: 21 January 2019

Accepted: 15 July 2019.

Study carried out in the Disciplina de Cirurgia Torácica, Instituto do Coração,

Faculdade de Medicina, Universidade de

São Paulo, São Paulo (SP) Brasil.

\section{INTRODUCTION}

In the last two decades, minimally invasive surgery has been established as the gold standard for the treatment of lung cancer. ${ }^{(1,2)}$ Several studies have been published and confirmed the role of video-assisted thoracoscopic surgery in complex resections such as lobectomies and pneumonectomies. ${ }^{(3,4)}$ Recently, robotic surgery has emerged as an alternative to conventional video-assisted thoracoscopic surgery, because it has the advantage of increasing the amplitude and precision of intracavitary maneuvers and movements, as well as improving visualization through three-dimensional imaging. ${ }^{(1)}$ The use of robotics in thoracic surgery has been established in studies that demonstrated its feasibility, especially for mediastinal tumors and pulmonary resections, such as pulmonary lobectomy and segmentectomies. ${ }^{(5-7)}$

Although the use of robotics in thoracic surgery is still in its early stages, it is promising. Large case series have shown good results regarding postoperative morbidity and mortality, as well as length of hospital stay and the need for readmission. ${ }^{(5-7)}$ Studies have shown the superiority of robotic surgery over traditional thoracotomy. (5) However, the concrete benefits of robotic surgery over conventional video-assisted thoracoscopic surgery are still the subject of study. ${ }^{(6,7)}$ In addition, the costs related to robotic surgery are still one of the determining factors in choosing the method. ${ }^{(8)}$

The financial issue related to robotic surgery is particularly relevant in low- and middle-income countries, where resources are limited. Therefore, an analysis of the cost-effectiveness of the use of new techniques is essential. In Brazil, the first robotic thoracic surgical procedures were performed in 2010. However, the first case series describing pulmonary resections was published in 2016 and essentially demonstrated the feasibility of the method. ${ }^{(1)}$ Nevertheless, data on the

Correspondence to:

Ricardo Mingarini Terra. Instituto do Coração, Hospital das Clínicas, FMUSP, Avenida Dr. Enéas de Carvalho Aguiar, 44, Bloco 2, $2^{\circ}$ andar, Sala 9, Cerqueira Cezar, CEP 05403-000, São Paulo, SP, Brasil.

Tel.: 5511 2661-5708. E-mail: rmterra@uol.com.br

Financial support: None. 
short- and medium-term results of this technique in our country are lacking. Therefore, the main objective of the present study was to describe the morbidity, mortality, and complete resection associated with robotic surgery for the treatment of non-small cell lung cancer in Brazil. The secondary objective was to report the rates of overall survival and disease-free survival in patients so treated.

\section{METHODS}

This was a retrospective study of patients diagnosed with non-small cell lung carcinoma and undergoing resection by robotic surgery between February of 2015 and July of 2018 by a group of thoracic surgeons at the São Paulo State Cancer Institute, Hospital Sírio-Libanês, Hospital Albert Einstein, Hospital Nove de Julho, and Hospital São Luiz, all located in the city of São Paulo, as well as the Hospital Copa Star/Rede D'Or, located in the city of Rio de Janeiro.

\section{Selection of patients and data collection}

We included all patients who underwent surgical resection of lung cancer by robotic surgery (segmentectomy, lobectomy, or bilobectomy) during the study period. Patients who underwent wedge surgical resection were excluded, as were those for whom the medical records were incomplete. Data were collected retrospectively through review of the electronic medical records available at all the hospitals. Data confidentiality and patient anonymity were preserved.

\section{Definition of outcomes}

Postoperative complications were defined as any complication that led to direct intervention involving treatment (pneumothorax, invasive procedures, etc.) or resulted in biochemical and diagnostic changes (e.g., to renal failure or subcutaneous emphysema). Complications were identified through the analysis of electronic medical records, laboratory tests, and imaging examinations.

Postoperative mortality was defined as death that occurred within the first 30 days after surgery. The survival rate was calculated based on the period from the date of surgery to the date of the last medical contact.

Surgical resection (completeness of the resection) was defined as complete, incomplete, or uncertain according to the criteria established by the International Society for the Study of Lung Cancer. ${ }^{(9)}$ In cases of segmentectomy, after removal of the specimen, routine analysis of the surgical margins was performed by frozen section biopsy.

\section{Surgical technique}

The first author participated in all the procedures, either as the surgeon operating the console or as the proctor. The surgical technique and treatment protocols were standardized and used in all cases. All patients underwent surgery with selective intubation confirmed by bronchoscopy. Although an epidural catheter for postoperative analgesia was used at the beginning of our study, ${ }^{(1)}$ it is no longer utilized. Anesthesia was carried out before the incision for the first port and, after the introduction of the camera, the 4th to 11th intercostal spaces were blocked under direct vision. The robotic lobectomy technique we used was originally described by Dylewski et al. ${ }^{(10)}$ and modified by our group. ${ }^{(1)}$ The patient is placed in the supine position with armpit padding, and the robot is positioned close to the head of the patient. In total there are four ports: three for the robotic arms and one for the assistant surgeon. (1) The first incision is performed at the 7th or 8th intercostal space in the posterior axillary line and, with the aid of an endoscope, the locations for the other ports are selected.

During the postoperative period, patients are usually moved to the inpatient unit. Only elderly patients with multiple comorbidities or those who had any intraoperative complications are moved to the ICU. Postoperative analgesia includes oral dipyrone every 6 $h$, as well as anti-inflammatory drugs and gabapentin. We avoided the use of oral opioids as much as possible.

\section{Statistical analysis}

Continuous variables are expressed as mean and standard deviation or as median and interquartile range (IQR). The Shapiro-Wilk test was used for normality analysis. Categorical variables are expressed as absolute and relative frequencies. Survival rate analyses were performed by using the Kaplan-Meier method. The type I error threshold was set at 5\%. Stata software, version 13 (StataCorp LP, College Station, TX, USA), was used for the statistical analyses.

\section{RESULTS}

We included 154 patients diagnosed with non-small cell lung cancer who underwent surgical resection by robotic surgery. The characteristics of the patients are described in Table 1 . The mean age was 65.0 \pm 9.5 years (range: $30-85$ years). As can be seen in Table 2, the most common histological type was adenocarcinoma, in 126 patients (82\%), followed by epidermoid carcinoma, in 14 (9\%), and carcinoid tumors, in 14 (9\%). Of the 154 patients in the sample, 109 (71\%) presented stage I lung cancer. The most common type of surgery was lobectomy, in 133 patients $(86.3 \%)$, followed by segmentectomy, in 21 (13.7\%), as shown in Table 3. The mean overall operative time was $209 \pm 80 \mathrm{~min}$; being $214 \pm 80 \mathrm{~min}$ for lobectomies and $167 \pm 51 \mathrm{~min}$ for segmentectomies $(p=0.01)$. The mean number of resected lymph nodes was 12.0 \pm 6.5 (range: $2-38$ ), and the mean number of lymph node stations sampled was $6 \pm 1$ (range: 2-9). The pathological outcome and final staging are described in Table 4. There was no conversion to video-assisted thoracoscopic surgery or thoracotomy in any case, nor was there significant intraoperative bleeding.

Postoperative complications occurred in 32 patients (20.4\%). Prolonged air leak was the most common 
Table 1. Characteristics of the sample. ${ }^{\mathrm{a}}$

\begin{tabular}{lc}
\hline \multicolumn{1}{c}{ Characteristic } & (N $=154)$ \\
Gender & $72(46.5)$ \\
Female & $82(53.5)$ \\
Male & $65 \pm 9.5(30-85)$ \\
Age, years & $27.1 \pm 4.9(19.6-51.4)$ \\
BMl, kg/m & \\
Pulmonary function & $2.35 \pm 0.74(1.04-4.29)$ \\
FEV ${ }_{1}$, L/min & $87 \pm 18(40-125)$ \\
FVC, \% of predicted & \\
Comorbidities & $91(62)$ \\
Smoking & $77(54)$ \\
Arterial hypertension & $34(24)$ \\
COPD & $33(23)$ \\
Previous neoplasia at another location & $26(18)$ \\
Heart failure & $20(14)$ \\
Diabetes mellitus & $6(4)$ \\
Kidney failure & $6(4)$ \\
Chronic liver disease &
\end{tabular}

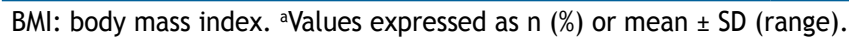

Table 2. Histological types.

\begin{tabular}{lc}
\multicolumn{1}{c}{ Histological type } & $(\mathbf{N}=\mathbf{1 5 4})$ \\
Adenocarcinoma in situ & 7 \\
Minimally invasive adenocarcinoma & 11 \\
Adenocarcinoma - predominantly lepidic & 11 \\
Adenocarcinoma - predominantly papillary & 10 \\
Adenocarcinoma - predominantly micropapillary & 4 \\
Adenocarcinoma - predominantly acinar & 22 \\
Adenocarcinoma - invasive mucinous pattern & 6 \\
Adenocarcinoma - enteral pattern & 3 \\
Adenocarcinoma - solid pattern & 2 \\
Invasive adenocarcinoma (subtype not reported) & 49 \\
Adenosquamous carcinoma & 1 \\
Typical carcinoid tumor & 11 \\
Atypical carcinoid tumor & 3 \\
Epidermoid carcinoma & 14 \\
\hline
\end{tabular}

complication, in 15 patients (9.5\%), and 7 patients (4.4\%) were discharged with a chest tube in place. In addition, 4 patients $(2.5 \%)$ required pleural drainage after removal of the chest tube. Chylothorax occurred in 4 patients $(2.5 \%)$. All were treated with dietary measures; however, 2 underwent reoperation for thoracic duct ligation due to persistent lymphatic fistula. One patient who underwent right upper lobectomy required reoperation due to torsion of the middle lobe, which was resected. Another patient had a severe anaphylactic reaction at the end of a segmentectomy, with subsequent cardiopulmonary arrest, which was promptly reversed, with no motor or neurological sequelae. Yet another patient presented trauma as a result of endotracheal intubation, leading to laryngeal edema and the need for tracheostomy during extubation. On postoperative day 7 , she presented intestinal perforation due to Ogilvie's syndrome.
Emergency laparotomy revealed a perforated cecum. A right hemicolectomy and temporary colostomy were performed. The patient showed no further complications in the postoperative period. The colostomy was reversed 2 months after hospital discharge. All of the complications are shown in Table 5.

The median length of hospital stay was 4 days (IQR: 3-6 days). The median pleural drainage time was 2 days (IQR: 2-4 days). Procedure-related death occurred in 1 patient $(0.5 \%), 12$ days after a lobectomy. That patient had developed pneumonia, sepsis, and multiple organ failure.

Pulmonary resection was categorized as complete in $97.4 \%$ of the cases. In $2.6 \%$ of patients, resection was categorized as uncertain due to the presence of at least one mediastinal lymph node affected by a neoplasm. Recurrence of neoplastic disease occurred in 11 patients $(7.8 \%)$. The median overall survival and 
Table 3. Types of surgical procedures performed.

\begin{tabular}{|c|c|}
\hline Type of surgical procedure & $(N=154)$ \\
\hline Lobectomy & $133(86.3 \%)$ \\
\hline Upper right lobe & 51 \\
\hline Upper right lobe + bronchoplasty & 2 \\
\hline Middle lobe & 5 \\
\hline Lower right lobe & 28 \\
\hline Upper left lobe & 21 \\
\hline Upper left lobe + bronchoplasty & 1 \\
\hline Lower left lobe & 24 \\
\hline Middle lobe + lower right lobe & 1 \\
\hline Segmentectomy & $21(13.7 \%)$ \\
\hline \multicolumn{2}{|l|}{ Upper right lobe } \\
\hline Apical segment (S1) & 1 \\
\hline Posterior segment (S2) & 2 \\
\hline Anterior segment (S3) & 1 \\
\hline \multicolumn{2}{|l|}{ Lower right lobe } \\
\hline Upper segment (S6) & 4 \\
\hline Basilar segments $(\mathrm{S} 7+\mathrm{S} 8+\mathrm{S} 9+\mathrm{S} 10)$ & 1 \\
\hline Segment $(S 7+S 8)$ & 1 \\
\hline \multicolumn{2}{|l|}{ Upper left lobe } \\
\hline Apical-posterior segments + anterior $(\mathrm{S} 1+\mathrm{S} 2+\mathrm{S} 3)$ & 6 \\
\hline Lingular segment (S4 + S5) & 1 \\
\hline Lingular segment (S4) & 1 \\
\hline \multicolumn{2}{|l|}{ Lower left lobe } \\
\hline Upper segment (S6) & 2 \\
\hline Anterior basal segment (S8) & 1 \\
\hline
\end{tabular}

Table 4. Final pathological tumor-node-metastasis staging.

\begin{tabular}{|c|c|c|c|c|c|}
\hline $\mathbf{T}$ & $\mathbf{N}$ & M & Stage & $\mathbf{n}$ & $\%$ \\
\hline In situ & 0 & 0 & & 7 & \\
\hline $1 \mathrm{a}(\mathrm{mi})$ & 0 & 0 & $1 \mathrm{~A} 1$ & 10 & 20.5 \\
\hline $1 \mathrm{a}$ & 0 & 0 & & 15 & \\
\hline $1 \mathrm{~b}$ & 0 & 0 & $1 \mathrm{~A} 2$ & 41 & 26.0 \\
\hline $1 c$ & 0 & 0 & $1 \mathrm{~A} 3$ & 22 & 14.0 \\
\hline $2 a$ & 0 & 0 & IB & 17 & 11.0 \\
\hline $2 b$ & 0 & 0 & IIA & 6 & 4.0 \\
\hline $1 b$ & 1 & 0 & & 5 & \\
\hline $1 c$ & 1 & 0 & IIB & 4 & 9.5 \\
\hline 3 & 0 & 0 & & 6 & \\
\hline $1 \mathrm{a}$ & 2 & 0 & & 2 & \\
\hline $1 b$ & 2 & 0 & IIIA & 4 & 11.5 \\
\hline $2 a$ & 2 & 0 & & 4 & \\
\hline $2 b$ & 2 & 0 & & 3 & \\
\hline 3 & 1 & 0 & & 3 & \\
\hline 4 & 1 & 0 & & 2 & \\
\hline 3 & 2 & 0 & IIIB & 4 & 2.5 \\
\hline $1 c$ & 0 & $1 b$ & IVA & 1 & 10 \\
\hline 3 & $x$ & $1^{\mathrm{a}}$ & IVA & 1 & 1.0 \\
\hline
\end{tabular}

T: tumor; N: node; and $\mathrm{M}$ : metastasis.

median recurrence-free survival are shown in Figure 1. Among patients with recurrence of neoplastic disease, 2 $(20 \%)$ had a confirmed tumor-node-metastasis (TNM) stage of N2. Disease recurrence occurred primarily in the pleura (35\%), as well as through metastasis to the central nervous system (15\%) and bones (10\%). The mean follow-up period was $326 \pm 274$ days (range: $3-1,110$ days). Two patients died due to the 
progression of neoplastic disease. The overall mortality rate in the present cohort was $1.5 \%$, corresponding to three deaths.

\section{DISCUSSION}

The present study portrays a consecutive series of 154 patients with primary lung cancer who were treated with pulmonary resection by robotic surgery. Our project started in $2015^{(1)}$ and has seen a large increase in the number of individuals so treated since then. Most of the patients had stage I lung cancer. This predominance of early-stage tumors is in contrast with data collected for Brazil as a whole. According to the registry of malignant neoplasms in the state of São Paulo, only 1,835 ( $8.8 \%$ ) of the 20,850 lung cancer patients registered between 2000 and 2010 had stage I disease. ${ }^{(11,12)}$ That difference can be explained by the fact that our sample was composed of highly selected patients treated surgically at public and private referral hospitals, where they had greater access to chest CT scans and lung cancer screening programs.

The morbidity and mortality rates in the present study were $20.4 \%$ and $0.5 \%$, respectively. These data are in line with those recently reported in the literature and represent the modern reality of thoracic surgery. This is a clear advance over the rates reported in studies previously conducted in Brazil. The analysis performed by Tsukazan et al., (3) using the database of the Sociedade Brasileira de Cirurgia Torácica (SBCT, Brazilian Society of Thoracic Surgery), compared patients submitted to pulmonary resection by thoracotomy and those submitted to pulmonary resection by video-assisted thoracoscopic surgery. The complication rate in the thoracotomy group was $30.1 \%$, compared with $21.8 \%$ in the video-assisted thoracoscopic surgery group. Regarding the mortality rate, it was $2.5 \%$ in the thoracotomy group and $1.6 \%$ in the video-assisted thoracoscopic surgery group. Another study using the
SBCT database, which included pulmonary resections by video-assisted thoracoscopic surgery, reported a surgical morbidity rate of $19.1 \%$ and a mortality rate of $2.0 \%$. ${ }^{(2)}$ In our study, morbidity is comparable to that in patients who underwent video-assisted thoracoscopic surgery. However, there was a significant reduction

(A)

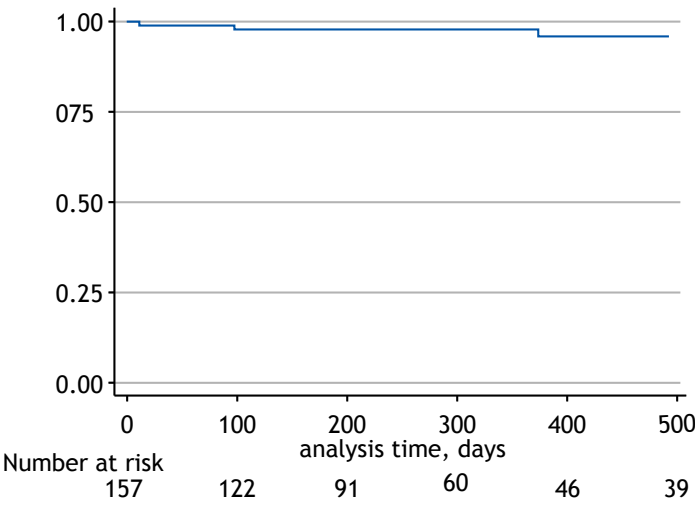

(B)

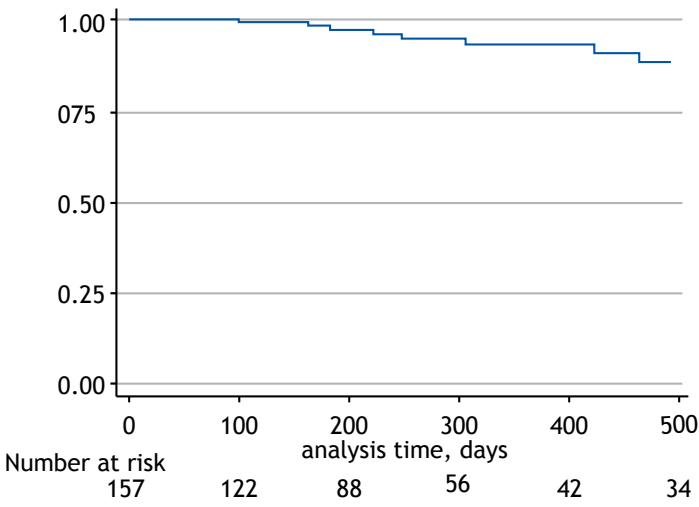

Figure 1. Kaplan-Meier curves. In $A$, the overall survival curve. In $B$, the disease-free survival curve.

Table 5. Complications. ${ }^{a}$

\begin{tabular}{lc}
\hline \multicolumn{1}{c}{ Complication } & $\mathbf{n}=32(20.4)$ \\
Prolonged air leak & $15(9.5)$ \\
Discharge with pleural drainage & $7(4.4)$ \\
Pneumothorax + further pleural drainage & $4(2.5)$ \\
Pulmonary embolism & $4(2.5)$ \\
Chylothorax & $4(2.5)$ \\
Pneumonia & $3(1.8)$ \\
Reoperation & $3(1.8)$ \\
Cardiac arrhythmia & $2(1.2)$ \\
Tracheostomy & $2(1.2)$ \\
Urinary tract infection & $2(1.2)$ \\
Non-dialysis dependent renal insufficiency & $2(1.2)$ \\
Dialysis dependent renal insufficiency & $1(0.6)$ \\
Endotracheal intubation trauma: glottic edema & $1(0.6)$ \\
Intestinal subocclusion & $1(0.6)$ \\
Intestinal perforation + colostomy & $1(0.6)$ \\
Hospital readmissions within 90 days & $12(7.5)$ \\
\hline
\end{tabular}

avalues expressed as $\mathrm{n}(\%)$ or mean \pm SD (variation). 
in postoperative mortality (1.6-2.0\% vs. $0.5 \%)$. This is corroborated by other studies of robotic pulmonary resections, which reported mortality rates of $0.2-1.3 \%$ and morbidity rates of $34.6-43.8 \% .^{(5-7)}$

Prolonged air leak was the most common complication in our sample. In fact, this finding is similar to that reported by other investigators. ${ }^{(5-7,13)}$ Air leak prolongs the length of hospital stay, increases the chance of pleural infection, and often leads to additional procedures such as chest tube insertion. In our study, 4 patients $(2.5 \%)$ required extended pleural drainage. In addition to the inconveniences associated with prolonged pleural drainage, prolonged hospitalization greatly increases hospital costs and decreases the cost-effectiveness of the robotic procedure. ${ }^{(14)}$

Although overall morbidity was in line with the most recent data, the profile of complications in our sample was different from that reported in other studies. Severe cardiovascular events occurred in only 3 patients. One patient had atrial fibrillation, which was treated with medications; another had myocardial ischemia and was treated with percutaneous coronary stenting; the third developed a severe anaphylactic reaction at the end of a segmentectomy, with subsequent cardiopulmonary arrest. Nasir et al. ${ }^{(15)}$ observed arrhythmia in $7 \%$ of patients who underwent pulmonary resection, a complication that was not observed in our sample. However, postoperative chylothorax was observed in 4 patients $(2.5 \%)$. We believe this is related to extensive lymph node dissection in the right paratracheal stations. These findings are confirmed in a study by Bryant et al., ${ }^{(16)}$ who demonstrated that lobectomy $(p=0.011)$, robotic surgery $(p=0.032)$ and the N2 pathological stage $(p=0.027)$ are predictors of chylothorax occurrence. Therefore, we currently recommend the use of clips in this region and initial aspiration of the pleural space at $-8 \mathrm{cmH}_{2} \mathrm{O}$ in the postoperative digital drainage system.

The survival rate curve for patients treated surgically in this study is above $90 \%$ at 2 years, which is consistent with data in the literature. ${ }^{(4-7)}$ However, because of the short follow-up period, it was not possible to calculate the median survival rate in our sample. With a longer follow-up period, we would be able to analyze the impact of sublobar resections among patients treated surgically, specifically in relation to the local recurrence rate. The rate of complete resection in the present study $(97.4 \%)$ is also consistent with data in the literature. ${ }^{(13,17)}$

Few studies have drawn direct comparisons between video-assisted thoracoscopic surgery and robotic surgery. However, there is evidence that robotic surgery is superior to traditional video-assisted thoracoscopic surgery. $(5,7,8,14,15)$ The former provides reductions in morbidity and especially operative mortality. In addition, the extent of the lymph node disease/dissection, and the resulting mediastinal upstaging, make robotic surgery more attractive in cases of locally advanced disease. (17) In the case of occult N2 disease, robotic surgery can also reduce recovery time and the time between surgery and adjunctive treatment, thereby making it more likely that full doses of systemic therapeutic agents will be administered to patients. ${ }^{(17)}$

We conclude that robotic pulmonary resection is safe for the treatment of lung cancer, and the initial experience in Brazil is comparable to that reported in studies conducted elsewhere. Certainly, the method and its indications will be consolidated over time.

\section{REFERENCES}

1. Terra RM, Araujo PH, Lauricella LL, Campos JR, Costa HF, PegoFernandes PM. Robotic pulmonary lobectomy for lung cancer treatment: program implementation and initial experience. J Bras Pneumol. 2016;42(3):185-90. https://doi.org/10.1590/S180637562015000000212

2. Terra RM, Kazantzis T, Pinto-Filho DR, Camargo SM, Martins-Neto $F$, Guimarães AN, et al. Anatomic pulmonary resection by videoassisted thoracoscopy: the Brazilian experience (VATS Brazil study). J Bras Pneumol. 2016:42(3):215-21. https://doi.org/10.1590/S180637562015000000337

3. Tsukazan MTR, Terra RM, Vigo Á, Fortunato GA, Camargo SM, de Oliveira HA, et al. Video-assisted thoracoscopic surgery yields bette outcomes than thoracotomy for anatomical lung resection in Brazil: a propensity score-matching analysis using the Brazilian Society of Thoracic Surgery database. Eur J Cardiothorac Surg. 2018;53(5):993998. https://doi.org/10.1093/ejcts/ezx442

4. Flores RM, Park BJ, Dycoco J, Aronova A, Hirth Y, Rizk NP et al. Lobectomy by video-assisted thoracic surgery (VATS) versus thoracotomy for lung cancer. J Thorac Cardiovasc Surg. 2009;138(1):11-8. https://doi.org/10.1016/j.jtcvs.2009.03.030

5. Louie BE, Wilson JL, Kim S, Cerfolio RJ, Park BJ, Farivar AS, Vallières E, Aye RW, Burfeind WR Jr, Block MI. Comparison of Video-Assisted Thoracoscopic Surgery and Robotic Approaches for Clinical Stage I and Stage II Non-Small Cell Lung Cancer Using The Society of Thoracic Surgeons Database. Ann Thorac Surg. 2016;102(3):917924. https://doi.org/10.1016/j.athoracsur.2016.03.032

6. Cerfolio RJ, Ghanim AF, Dylewski M, Veronesi G, Spaggiari L, Park BJ. The long-term survival of robotic lobectomy for non-small cell lung cancer: A multi-institutional study. J Thorac Cardiovasc Surg 2018;155(2):778-786. https://doi.org/10.1016/j.jtcvs.2017.09.016

7. Oh DS, Reddy RM, Gorrepati ML, Mehendale S, Reed MF. RoboticAssisted, Video-Assisted Thoracoscopic and Open Lobectomy: Propensity-Matched Analysis of Recent Premier Data. Ann Thorac Surg. 2017;104(5):1733-1740. https://doi.org/10.1016/j. athoracsur.2017.06.020

8. Swanson SJ, Miller DL, McKenna RJ Jr, Howington J, Marshall MB Yoo AC, et al. Comparing robot-assisted thoracic surgical lobectomy with conventional video-assisted thoracic surgical lobectomy and wedge resection: results from a multihospital database (Premier) J Thorac Cardiovasc Surg. 2014;147(3):929-37. https://doi. org/10.1016/j.jtcvs.2013.09.046

9. Rami-Porta R, Wittekind C, Goldstraw P: International Association for the Study of Lung Cancer (IASLC) Staging Committee. Complete resection in lung cancer surgery: proposed definition. Lung Cancer. 2005;49(1):25-33. https://doi.org/10.1016/j.lungcan.2005.01.001

10. Dylewski MR, Ohaeto AC, Pereira JF. Pulmonary resection using a total endoscopic robotic video-assisted approach. Semin Thorac Cardiovasc Surg. 2011;23(1):36-42. https://doi.org/10.1053/j. semtcvs.2011.01.005

11. Araujo LH, Baldotto C, Castro G Jr, Katz A, Ferreira CG, Mathias $C$, et al. Lung cancer in Brazil. J Bras Pneumol. 2018;44(1):55-64. https:// doi.org/10.1590/s1806-37562017000000135

12. Secretaria de Estado da Saúde de São Paulo. Fundação Oncocentro de São Paulo [homepage on the Internet]. São Paulo: a Fundação [cited 2015 Oct 1]. Acesso ao TABNET. Available from: http://fosp. saude.sp.gov.br/publicacoes/tabnet 
13. Casiraghi M, Galetta D, Borri A, Tessitore A, Romano R, Diotti C, et al. Ten Years' Experience in Robotic-Assisted Thoracic Surgery for Early Stage Lung Cancer. Thorac Cardiovasc Surg. 2018 Apr 1. [Epub ahead of print]

14. Kneuertz PJ, Singer E, D'Souza DM, Moffatt-Bruce SD, Merritt RE. Postoperative complications decrease the cost-effectiveness of robotic-assisted lobectomy. Surgery. 2019;165(2):455-460. https:// doi.org/10.1016/j.surg.2018.08.024

15. Nasir BS, Bryant AS, Minnich DJ, Wei B, Cerfolio RJ. Performing robotic lobectomy and segmentectomy: cost, profitability, and outcomes. Ann Thorac Surg. 2014;98(1):203-8; discussion 208-9. https://doi.org/10.1016/j.athoracsur.2014.02.051

16. Bryant AS, Minnich DJ, Wei B, Cerfolio RJ. The incidence and management of postoperative chylothorax after pulmonary resection and thoracic mediastinal lymph node dissection. Ann Thorac Surg. 2014;98(1):232-5; discussion 235-7. https://doi.org/10.1016/j. athoracsur.2014.03.003

17. Veronesi $G$, Novellis $P$, Voulaz E, Alloisio M. Robot-assisted surgery for lung cancer: State of the art and perspectives. Lung Cancer. 2016;101:28-34. https://doi.org/10.1016/j.lungcan.2016.09.004 